Access to this work was provided by the University of Maryland, Baltimore County (UMBC) ScholarWorks@UMBC digital repository on the Maryland Shared Open Access (MD-SOAR) platform.

Please provide feedback

Please support the ScholarWorks@UMBC repository by emailing scholarworks-group@umbc.edu and telling us what having access to this work means to you and why it's important to you. Thank you. 


\title{
Adaptive Coarse Space Selection in the BDDC and the FETI-DP Iterative Substructuring Methods: Optimal Face Degrees of Freedom
}

\author{
Jan Mandel ${ }^{1 \star}$ and Bedřich Sousedík ${ }^{2 \star \star}$ \\ 1 Department of Mathematics, University of Colorado at Denver, P.O. Box 173364, \\ Campus Box 170, Denver, CO 80217, USA. jmandel@math.cudenver.edu \\ 2 Department of Mathematics, Faculty of Civil Engineering, Czech Technical \\ University in Prague, Thákurova 7, 16636 Prague 6, Czech Republic. \\ bedrich.sousedik@fsv.cvut.cz
}

Summary. We propose adaptive selection of the coarse space of the BDDC and FETI-DP iterative substructuring methods by adding coarse degrees of freedom (dofs) on faces between substructures constructed using eigenvectors associated with the faces. Provably the minimal number of coarse dofs on the faces is added to decrease a heuristic indicator of the condition number under a target value specified a priori. It is assumed that corner dofs are already sufficient to prevent relative rigid body motions of any two substructures with a common face. It is shown numerically on a $2 \mathrm{D}$ elasticity problem that the indicator is reasonably close to the actual condition number and that the method can find automatically the hard part of the problem and concentrate the computational work there to achieve the target value for the condition number and good convergence of the iterations, at a modest cost.

\subsection{Introduction}

The BDDC and FETI-DP methods are iterative substructuring methods that use coarse degrees of freedom associated with corners and edges (in 2D) or faces (in 3D, further on just faces) between substructures, and they are currently the most advanced versions of the BDD and FETI families of methods. The BDDC method [2] is a Neumann-Neumann method of Schwarz type [3]. The BDDC method iterates on the system of primal variables reduced to the interfaces between the substructures and it can be understood as further development of the BDD method [10]. The FETI-DP method [4, 5] is a dual method that iterates on a system for Lagrange multipliers that enforce

\footnotetext{
* Supported under a contract with Sandia National Laboratories.

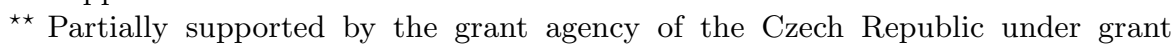
106/05/2731 and Program of the Information Society 1ET400300415.
} 
continuity on the interfaces. Algebraic relations between FETI and BDD methods were pointed out in $[6,7,12]$. A common bound on the condition number of both the FETI and the BDD method in terms of a single inequality was given in [7]. In the case of corner constraints only, methods same as BDDC were derived as primal versions of FETI-DP in $[1,6]$. In [11], it was proved that the eigenvalues of BDDC and FETI-DP are identical and a bound on the condition number was obtained in terms of matrix data only.

In this contribution, we show how to use the algebraic estimate of the condition number from [11] to develop an adaptive fast method. First we estimate the condition number as the solution of an eigenvalue problem, then obtain a reliable heuristic indicator from the eigenvalues for two substructures with a common face faces. Finally, we show how to use the eigenvectors to obtain coarse degrees of freedom that result in an optimal decrease of the indicator. We demonstrate on numerical examples that the indicator is quite close that such an adaptive approach results in the concentration of computational work in a small part of the problem, leading to good convergence behavior at a small added cost.

Related work on adaptive coarse space selection has focused on the global problem of selecting the smallest number of corners to prevent coarse mechanisms [9] and the smallest number of coarse degrees of freedom to assure asymptotically optimal convergence estimates [8]. In contrast, our indicator of condition number is local in nature and we assume that corner degrees of freedom are already sufficient to prevent relative rigid body motions of any two substructures with a common face.

\subsection{Formulation of BDDC and FETI-DP}

We need to briefly recall the formulation of the methods and the condition number bound. Let $K_{s}$ be the stiffness matrix and $v_{s}$ the vector of degrees of freedom (dofs) for substructure $s$. We want to solve the problem in decomposed form

$$
\frac{1}{2} v^{T} K v-v^{T} f \rightarrow \min , \quad v=\left[\begin{array}{c}
v_{1} \\
\vdots \\
v_{N}
\end{array}\right] \quad K=\left[\begin{array}{ccc}
K_{1} & & \\
& \ddots & \\
& & K_{N}
\end{array}\right]
$$

subject to continuity dofs between substructures. Partitioning the dofs in each subdomain $s$ into internal and interface (boundary)

$$
K_{s}=\left[\begin{array}{cc}
K_{s}^{i i} & K_{s}^{i b} \\
K_{s}^{i b T} & K_{s}^{b b}
\end{array}\right], \quad v_{s}=\left[\begin{array}{c}
v_{s}^{i} \\
v_{s}^{b}
\end{array}\right], \quad f_{s}=\left[\begin{array}{c}
f_{s}^{i} \\
f_{s}^{b}
\end{array}\right]
$$

and eliminating the interior dofs we obtain the problem reduced to interfaces

$$
\frac{1}{2} w^{T} S w-w^{T} g \rightarrow \min , \quad S=\operatorname{diag}\left(S_{s}\right), \quad S_{s}=K_{s}^{b b}-K_{s}^{i b^{T}} K_{s}^{i i^{-1}} K_{s}^{i b},
$$


again subject to continuity of dofs between substructures.

In BDD type methods, the continuity of dofs between substructures is enforced by imposing common values on substructures interfaces: $w=R u$ for some $u$, where

$$
R=\left[\begin{array}{c}
R_{1} \\
\vdots \\
R_{N}
\end{array}\right]
$$

and $R_{s}$ is the operator of restriction of global dofs on the interfaces to substructure $s$. In FETI type methods, continuity of dofs between substructures is enforced by the constraint $B w=0$, where the entries of $B$ are typically $0, \pm 1$. By construction, we have $R_{s} R_{s}^{T}=I$ and range $R=\operatorname{null} B$.

Node is the set of all dofs associated with the same location in space. Nodes such that no other node is adjacent to the same set of substructures are called corners. Face is the set of all dofs shared by two substructures that contains more than one node.

A BDDC or FETI-DP method is specified by the choice of coarse dofs and the choice of weights for intersubdomain averaging. To define the coarse problem for BDDC, choose a matrix $Q_{P}^{T}$ that selects coarse dofs $u_{c}$ from global interface dofs $u$, e.g. as values at corners or averages on faces:

$$
u_{c}=Q_{P}^{T} u .
$$

The space $\widetilde{W}$ will consist of all vectors of substructure interface dofs such that the coarse dofs are continuous between substructures,

$$
\widetilde{W}=\left\{w \in W: \exists u_{c} \forall s: C_{s} w_{s}=R_{c s} u_{c}\right\}
$$

where $C_{s}=R_{c s} Q_{P}^{T} R_{s}^{T}$ maps a collection of substructure dofs to a collection of coarse dofs on substructure $s$, and $R_{c s}$ restricts a vector of all coarse dof values into a vector of coarse dof values that can be nonzero on substructure $s$. The dual approach in FETI-DP is to construct $Q_{D}$ such that $\widetilde{W}=\operatorname{null} Q_{D}^{T} B$.

In BDDC, the intersubdomain averaging is defined by the matrices $D_{P}=\operatorname{diag}\left(D_{P s}\right)$ that form a decomposition of unity, $R^{T} D_{P} R=I$. The corresponding dual matrices in FETI-DP are $B_{D}=\left[D_{D 1} B_{1}, \ldots D_{D N} B_{N}\right]$, where the dual weights $D_{D s}$ are defined so that $B_{D}^{T} B+R R^{T} D_{P}=I$.

The BDDC method is then the method of conjugate gradients for the assembled system $A u=R^{T} g$ with the system matrix $A=R^{T} S R$ and the preconditioner $P$ defined by $\operatorname{Pr}=R^{T} D_{P}\left(\Psi u_{c}+z\right)$, where $u_{c}$ is the solution of the coarse problem $\Psi^{T} S \Psi u_{c}=\Psi^{T} D_{P}^{T} R r$ and $z$ is the solution of

$$
\begin{aligned}
S z+C^{T} \mu & =D_{P}^{T} R r \\
C z & =
\end{aligned}
$$

which is a collection of independent substructure problems. The coarse basis functions $\Psi$ are defined by energy minimization, 


$$
\left[\begin{array}{cc}
S & C^{T} \\
C & 0
\end{array}\right]\left[\begin{array}{l}
\Psi \\
\Lambda
\end{array}\right]=\left[\begin{array}{c}
0 \\
R_{c}
\end{array}\right]
$$

The FETI-DP method solves the saddle point problem

$$
\min _{w \in \widetilde{W}} \max _{\lambda} \mathcal{L}(w, \lambda)=\max _{\lambda} \min _{w \in \widetilde{W}} \mathcal{L}(w, \lambda),
$$

where $\mathcal{L}(w, \lambda)=\frac{1}{2} w^{T} S w-w^{T} f+w^{T} B^{T} \lambda$ by iterating on the dual problem $\frac{\partial \mathcal{F}(\lambda)}{\partial \lambda}=F \lambda-h=0$, where

$$
\mathcal{F}(\lambda)=\min _{w \in \widetilde{W}} \mathcal{L}(w, \lambda),
$$

by conjugate gradients with the preconditioner $M=B_{D} S B_{D}^{T}$. See [11] for more details.

\subsection{Indicator of the Condition Number}

Theorem 1 ([11]). The eigenvalues of the preconditioned operators $P A$ of $B D D C$ and $M F$ of FETI-DP are same except for eigenvalues of zero and one, and the condition numbers satisfy

$$
\kappa_{\mathrm{BDDC}}=\kappa_{\mathrm{FETI}-\mathrm{DP}} \leq \omega=\sup _{w \in \widetilde{W}} J(\omega), \quad J(\omega)=\frac{\left\|B_{D}^{T} B w\right\|_{S}^{2}}{\|w\|_{S}^{2}} .
$$

Here, the condition number is the ratio of the largest and the smallest nonzero eigenvalue. Zero eigenvalues in FETI-DP are caused by redundant constraints, common in practice.

As an indicator of the condition number, we propose the maximum of the bounds from Theorem 1 computed by considering only one pair of adjacent substructures $s, t$ with a common face at a time:

$$
\omega \approx \widetilde{\omega}=\max _{s t} \omega_{s t}, \quad \omega_{s t}=\sup _{w_{s t} \in \widetilde{W}_{s t}} J_{s t}\left(w_{s t}\right) .
$$

All quantities with the subscript ${ }_{s t}$ are the same as without the subscript but defined using the domain consisting of the substructures $s$ and $t$ only.

Theorem 2. Let $a>0, \Pi_{s t}$ be the orthogonal projection onto $\widetilde{W}_{s t}$, and $I-\bar{\Pi}_{s t}$ be the orthogonal projection onto

$$
\operatorname{null}\left(\Pi_{s t} S_{s t} \Pi_{s t}+a\left(I-\Pi_{s t}\right)\right) .
$$

Then the stationary values $\omega_{s t, 1} \geq \omega_{s t, 2} \geq \ldots$ and the corresponding stationary vectors $w_{s t, k}$ of the Rayleigh quotient $J_{\text {st }}$ on $\widetilde{W}_{\text {st }}$ satisfy 


$$
X_{s t} w_{s t, k}=\omega_{s t, k} Y_{s t} w_{s t, k}
$$

with $Y_{\text {st }}$ positive definite, where

$$
\begin{aligned}
X_{s t} & =\Pi_{s t} B_{s t}^{T} B_{D s t} S_{s t} B_{D s t}^{T} B_{s t} \Pi_{s t}, \\
Y_{s t} & =\left(\bar{\Pi}_{s t}\left(\Pi_{s t} S_{s t} \Pi_{s t}+a\left(I-\Pi_{s t}\right)\right) \bar{\Pi}_{s t}+a\left(I-\bar{\Pi}_{s t}\right)\right)
\end{aligned}
$$

The eigenvalue problem (1.2) is obtained by projecting the gradient of the Rayleigh quotient $J_{s t}\left(w_{s t}\right)$ onto the complement in $\widetilde{W}_{s t}$ of the subspace where its denominator $\left\|w_{s t}\right\|_{S_{s t}}^{2}=0$, in two steps. Both projections $\Pi_{s t}$ and $\bar{\Pi}_{s t}$ are computed by matrix algebra, which is straightforward to implement numerically. The computation of $\Pi_{s t}$ involves minimization with Lagrange multipliers for the condition that the values of the coarse dofs on the the substructures $s$ and $t$ coincide. The projection $I-\bar{\Pi}_{s t}$ is onto a subspace of null $S_{s t}$, and it is easily constructed computationally if a matrix $Z_{s t}$ is given such that null $S_{s t} \subset$ range $Z_{s t}$. The matrix $Z_{s t}$ with columns consisting of the coarse basis functions can be used because the span of the coarse basis functions contains the rigid body modes. However, often the rigid body modes are available directly, which leads to a smaller matrix $Z_{s t}$ and thus cheaper computation. Since $Y_{s t}$ is positive definite, its Choleski decomposition exists, and we reduce (1.2) a symmetric eigenvalue problem, which is easier and more efficient to solve numerically.

\subsection{Optimal Coarse Degrees of Freedom on Faces}

Writing $\widetilde{W}_{s t}$ in the dual form $\widetilde{W}_{s t}=\operatorname{null} Q_{D s t}^{T} B_{s t}$ suggests how to add coarse dofs in an optimal way to decrease the value of indicator $\widetilde{\omega}$. The following theorem follows immediately from the standard characterization of eigenvalues as minima and maxima of the Rayleigh quotient on subspaces spanned by eigvectors, applied to (1.2).

Theorem 3. Suppose $\ell_{s t} \geq 0$ and the dual coarse dof selection matrix $Q_{D s t}^{T}$ is augmented to become $\left[Q_{D s t}^{T}, q_{D s t, 1}^{T}, \ldots, q_{D s t, \ell_{s t}}^{T}\right]$ with $q_{D s t, k}^{T}=$ $w_{s t, k}^{T} B_{s t}^{T} B_{D s t} S_{s t} B_{D s t}^{T}$, where $w_{s t, k}^{T}$ are the eigenvectors from (1.2). Then $\omega_{s t}=\omega_{s t, \ell_{s t}+1}$, and $\omega_{s t} \geq \omega_{s t, \ell_{s t}+1}$ for any other augmentation of $Q_{D s t}^{T}$ by at most $\ell_{\text {st }}$ columns.

In particular, if $\omega_{s t, \ell_{s t}+1} \leq \tau$ for all pairs of substructures $s, t$ with a common face, then $\widetilde{\omega} \leq \tau$.

Theorem 3 allows us to guarantee that the condition number indicator $\widetilde{\omega} \leq \tau$ for a given target value $\tau$, by adding the smallest possible number of face coarse dofs.

The primal coarse space selection mechanism that corresponds to this augmentation can be seen easily in the case when the entries of $B_{s t}$ are +1 


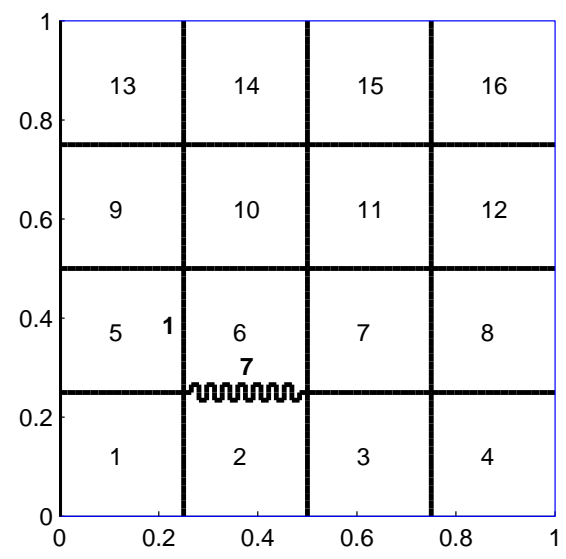

Fig. 1.1. Mesh with $H / h=16,4 \times 4$ substructures, and one jagged edge between substructures 2 and 6 . Zero displacement is imposed on the left edge. For compressible elasticity (Tables 1.1 and 1.2(a)) and tolerance $\tau=10,7$ coarse dofs at the jagged edge and 1 coarse dof at an adjacent edge are added automatically.

for substructure $s$ and -1 for substructrure $t$. Then $w_{s t} \in \widetilde{W}_{s t}$ can be written as

$$
Q_{D s t}^{T}\left(I_{s t} w_{s}-I_{t s} w_{t}\right)=0
$$

where $I_{s t}$ is the $0-1$ matrix that selects from $w_{s}$ the degrees of freedom on the intersection of the substructures $s$ and $t$. Each column of $q_{D}$ of $Q_{D s t}$ defines a coarse degree of freedom associated with the interface of substructures $s$ and $t$. The corresponding column $q_{P}$ of $Q_{P}$ is such that

$$
q_{P}^{T} R_{s}^{T}=q_{D}^{T} I_{s t}
$$

Because $R_{s}$ is also a $0-1$ matrix, this means that the vector $q_{P}$ is formed by a scattering of the entries of the vector $q_{D}$.

\subsection{Numerical Results}

Consider plane elasticity discretized by bilinear elements on a rectangular mesh decomposed into 16 substructure, with one single edge between substructures being jagged (Fig. 1.1). We have computed the eigenvalues and eigenvectors of (1.2) by setting up the matrices and using standard methods for the symmetric eigenvalue problem in Matlab. The eigenvalues $\omega_{s t, k}$ associated with edges between substructures (Table 1.1) clearly distinguish between the single problematic edge and the others. Adding the coarse dofs created from the associated eigenvectors according to Theorem 3 decreases 


\begin{tabular}{r|r|r|r|r|r|r|r|r|r}
$s$ & $t$ & $\omega_{s t, 1}$ & $\omega_{s t, 2}$ & $\omega_{s t, 3}$ & $\omega_{s t, 4}$ & $\omega_{s t, 5}$ & $\omega_{s t, 6}$ & $\omega_{s t, 7}$ & $\omega_{s t, 8}$ \\
\hline 1 & 2 & 3.7 & 2.3 & 1.4 & 1.3 & 1.1 & 1.1 & 1.1 & 1.1 \\
1 & 5 & 5.8 & 3.2 & 2.3 & 1.4 & 1.2 & 1.1 & 1.1 & 1.1 \\
2 & 3 & 6.0 & 2.5 & 1.7 & 1.3 & 1.2 & 1.1 & 1. & 1.1 \\
2 & 6 & 21.7 & 19.5 & 17.8 & 14.9 & 14.5 & 11.7 & 11.2 & 9.7 \\
3 & 4 & 3.3 & 2.3 & 1.4 & 1.3 & 1.1 & 1.1 & 1.1 & 1.1 \\
3 & 7 & 7.1 & 5.1 & 3.2 & 1.8 & 1.4 & 1.3 & 1.2 & 1.1 \\
4 & 8 & 5.9 & 3.4 & 2.6 & 1.4 & 1.2 & 1.1 & 1.1 & 1.1 \\
5 & 6 & 12.0 & 4.9 & 4.4 & 1.8 & 1.6 & 1.3 & 1.3 & 1.2 \\
5 & 9 & 5.9 & 3.4 & 2.6 & 1.4 & 1.3 & 1.3 & 1.1 & 1.1 \\
6 & 7 & 8.7 & 4.9 & 3.9 & 1.8 & 1.5 & 1.3 & 1.2 & 1.1 \\
6 & 10 & 7.3 & 4.8 & 3.4 & 1.8 & 1.4 & 1.3 & 1.2 & 1.1
\end{tabular}

Table 1.1. Several largest eigenvalues $\omega_{s t, k}$ for several edges for the elasticity problem from Fig. 1.1 with $H / h=16 .(s, t)=(2,6)$ is the jagged edge.

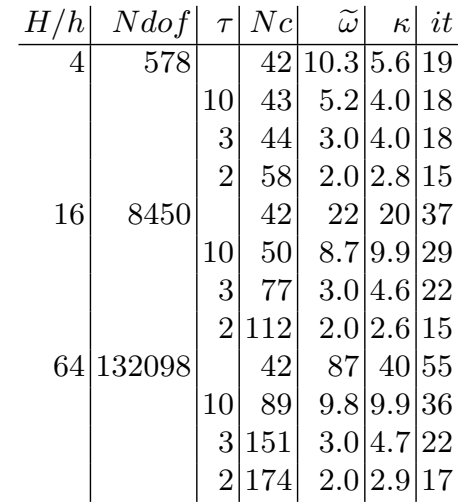

(a) compressible elasticity

\begin{tabular}{r|r|r|r|r|r|r}
$H / h$ & $N d o f$ & $\tau$ & $N c$ & $\widetilde{\omega}$ & $\kappa$ & $i t$ \\
\hline 4 & 578 & & 42 & 285 & 208 & 64 \\
& & 10 & 68 & 8.0 & 8.6 & 28 \\
& & 3 & 89 & 2.9 & 4.6 & 22 \\
16 & & 2450 & 114 & 2.0 & 2.6 & 16 \\
& & 42 & 1012 & 1010 & 161 \\
& & 87 & 9.8 & 9.9 & 29 \\
& & 3 & 77 & 3.0 & 4.6 & 22 \\
& 2 & 126 & 2.0 & 2.9 & 19 \\
& 132098 & 42 & 6910 & NA & $\infty$ \\
& & 10 & 183 & 9.8 & 9.7 & 37 \\
& 3 & 213 & 3.0 & 4.9 & 26 \\
& 2 & 274 & 2.0 & 3.0 & 20
\end{tabular}

(b) almost incompressible

Table 1.2. BDDC for plane elasticity on a square with one jagged edge. The Lamé coefficients are $\lambda=1$ and $\mu=2$ for (a), and $\lambda=1000$ and $\mu=2$ for (b). $H / h$ is the number of elements per substructure in one direction, $N d o f$ the number of dofs in the problem, $\tau$ the condition number tolerance as in Theorem 3, Nc the number of coarse dofs, $\widetilde{\omega}$ the value of the condition number indicator from $(1.1), \kappa$ the approximate condition number from the Lanczos sequence in conjugate gradients, and $i t$ the number of BDDC iterations for relative residual tolerance $10^{-8}$.

the value of the condition number indicator $\widetilde{\omega}$ and improves convergence at the cost of increasing the number of coarse dofs. This effect is even more pronounced for almost incompressible elasticity where the iterations converge poorly or not at all without the additional coarse dofs. This incompressible elasticity problem is particularly hard for an iterative method because standard bilinear elements were used instead of stable elements or reduced integration. In all cases, values of the condition number indicator $\widetilde{\omega}$ are quite close to the actually observed condition numbers $\kappa$ (Table 1.2). 


\section{References}

1. J.-M. Cros. A preconditioner for the Schur complement domain decomposition method. In Ismael Herrera, David E. Keyes, and Olof B. Widlund, editors, Domain Decomposition Methods in Science and Engineering, pages 373-380. National Autonomous University of Mexico (UNAM), México, 2003. 14th International Conference on Domain Decomposition Methods, Cocoyoc, Mexico, January 6-12, 2002.

2. Clark R. Dohrmann. A preconditioner for substructuring based on constrained energy minimization. SIAM J. Sci. Comput., 25(1):246-258, 2003.

3. Maksymilian Dryja and Olof B. Widlund. Schwarz methods of NeumannNeumann type for three-dimensional elliptic finite element problems. Comm. Pure Appl. Math., 48(2):121-155, 1995.

4. Charbel Farhat, Michael Lesoinne, and Kendall Pierson. A scalable dualprimal domain decomposition method. Numer. Linear Algebra Appl., 7:687-714, 2000. Preconditioning techniques for large sparse matrix problems in industrial applications (Minneapolis, MN, 1999).

5. Charbel Farhat, Michel Lesoinne, Patrick LeTallec, Kendall Pierson, and Daniel Rixen. FETI-DP: a dual-primal unified FETI method. I. A faster alternative to the two-level FETI method. Internat. J. Numer. Methods Engrg., 50(7):15231544, 2001.

6. Yannis Fragakis and Manolis Papadrakakis. The mosaic of high performance domain decomposition methods for structural mechanics: Formulation, interrelation and numerical efficiency of primal and dual methods. Comput. Methods Appl. Mech. Engrg., 192:3799-3830, 2003.

7. Axel Klawonn and Olof B. Widlund. FETI and Neumann-Neumann iterative substructuring methods: connections and new results. Comm. Pure Appl. Math., 54(1):57-90, 2001.

8. Axel Klawonn and Olof B. Widlund. Selecting constraints in dual-primal FETI methods for elasticity in three dimensions. In R. Kornhuber, R. Hoppe, J. Priaux, O. Pironneau, O. Widlund, and J. Xu, editors, Domain Decomposition Methods in Science and Engineering, Lecture Notes in Computational Science and Engineering, pages 67-81. Springer, 2004. Proceedings of the 15th International Conference on Domain Decomposition Methods in Berlin, Germany, 2003.

9. M. Lesoinne. A FETI-DP corner selection algorithm for three-dimensional problems. In Ismael Herrera, David E. Keyes, and Olof B. Widlund, editors, Domain Decomposition Methods in Science and Engineering, pages 217-223. National Autonomous University of Mexico (UNAM), México, 2003. 14th International Conference on Domain Decomposition Methods, Cocoyoc, Mexico, January 6-12, 2002.

10. Jan Mandel. Balancing domain decomposition. Comm. Numer. Methods Engrg., 9(3):233-241, 1993.

11. Jan Mandel, Clark R. Dohrmann, and Radek Tezaur. An algebraic theory for primal and dual substructuring methods by constraints. Appl. Numer. Math., 54:167-193, 2005.

12. Daniel J. Rixen, Charbel Farhat, Radek Tezaur, and Jan Mandel. Theoretical comparison of the FETI and algebraically partitioned FETI methods, and performance comparisons with a direct sparse solver. Int. J. Numer. Meth. Engrg, 46:501-534, 1999. 
